\title{
ARTICLE
}

\section{A high-density, multi-parental SNP genetic map on apple validates a new mapping approach for outcrossing species}

\author{
Erica A Di Pierro ${ }^{1}$, Luca Gianfranceschi ${ }^{1}$, Mario Di Guardo ${ }^{2,3}$, Herma JJ Koehorst-van Putten ${ }^{2}$, Johannes W Kruisselbrink ${ }^{4}$, Sara Longhi ${ }^{2}$, \\ Michela Troggio ${ }^{3}$, Luca Bianco ${ }^{3}$, Hélène Muranty ${ }^{5}$, Giulia Pagliarani ${ }^{6}$, Stefano Tartarini ${ }^{6}$, Thomas Letschka ${ }^{7}$, Lidia Lozano Luis ${ }^{7}$, \\ Larisa Garkava-Gustavsson ${ }^{8}$, Diego Micheletti ${ }^{3}$, Marco CAM Bink ${ }^{4,9}$, Roeland E Voorrips ${ }^{2}$, Ebrahimi Aziz ${ }^{2}$, Riccardo Velasco ${ }^{3}$, \\ François Laurens ${ }^{5}$ and W Eric van de Weg $^{2}$
}

Quantitative trait loci (QTL) mapping approaches rely on the correct ordering of molecular markers along the chromosomes, which can be obtained from genetic linkage maps or a reference genome sequence. For apple (Malus domestica Borkh), the genome sequence $v 1$ and $v 2$ could not meet this need; therefore, a novel approach was devised to develop a dense genetic linkage map, providing the most reliable marker-loci order for the highest possible number of markers. The approach was based on four strategies: (i) the use of multiple full-sib families, (ii) the reduction of missing information through the use of HaploBlocks and alternative calling procedures for single-nucleotide polymorphism (SNP) markers, (iii) the construction of a single backcross-type data set including all families, and (iv) a two-step map generation procedure based on the sequential inclusion of markers. The map comprises 15417 SNP markers, clustered in $3 \mathrm{~K}$ HaploBlock markers spanning $1267 \mathrm{cM}$, with an average distance between adjacent markers of $0.37 \mathrm{cM}$ and a maximum distance of $3.29 \mathrm{cM}$. Moreover, chromosome 5 was oriented according to its homoeologous chromosome 10. This map was useful to improve the apple genome sequence, design the Axiom Apple $480 \mathrm{~K}$ SNP array and perform multifamily-based QTL studies. Its collinearity with the genome sequences v1 and v3 are reported. To our knowledge, this is the shortest published SNP map in apple, while including the largest number of markers, families and individuals. This result validates our methodology, proving its value for the construction of integrated linkage maps for any outbreeding species.

Horticulture Research (2016) 3, 16057; doi:10.1038/hortres.2016.57; Published online 23 November 2016

\section{INTRODUCTION}

Genetic linkage maps play a major role in clarifying the genetic control of important traits and the development of DNA-based diagnostic tools for marker-assisted breeding. They are supposed to reflect the order of genes and molecular markers as they occur on the chromosomes and are critical resources for: (i) the identification of gene location on chromosomes via quantitative trait loci (QTL) discovery studies, ${ }^{1-3}$ (ii) the building of reference genome sequences through anchoring, ordering and orienting of contigs and scaffolds, ${ }^{4}$ and (iii) the cloning of genes through mapbased approaches. ${ }^{5-7}$ Most of the economically important traits in plant breeding, such as yield and product quality, are quantitative and controlled by multiple genes. Therefore, identifying the genomic location of such genes is a high priority for selecting new improved crop varieties. ${ }^{8,9}$ Remarkable advances have been achieved in understanding the functional complexity underpinning quantitative traits. A number of QTL with strong effects on phenotypic variation have been discovered, genetically positioned, validated and, in various cases, successfully exploited in marker-assisted breeding. ${ }^{9-11}$

In outbreeding species, conventional QTL discovery approaches rely on the availability of genetic linkage maps and segregating bi-parental full-sib (FS) families. However, a single FS family is unlikely to segregate for all QTL, thus providing only partial information. Currently, QTL mapping is shifting toward the simultaneous analysis of more complex pedigreed FS-families, derived by multiple direct parents and founders. ${ }^{12-18}$ This approach increases the probability of detecting QTL and capturing allelic variation while it improves the characterization of QTL performance in different genetic backgrounds. ${ }^{12,19-21}$

The EU-funded FruitBreedomics project ${ }^{10}$ was aimed, among other objectives, to clarify the genetic determination of a series of fruit quality traits in apple through a multifamily QTL mapping approach using molecular markers from a $20 \mathrm{~K}$ Infinium SNP array. ${ }^{22}$ This raised the need for a reference genetic linkage map allowing adequate integration of SNP marker data across wide germplasm. The accurateness of marker order is crucial to remove sources of spurious double recombinants and to narrow the intervals where QTL are located. When a high-quality consensus map or reference genome sequence is available, they can be used for the correct ordering of markers.

At the onset of this work, various genetic linkage maps were available for apple with most based on a single FS family ${ }^{2,23-32}$ and some based on a few FS families. ${ }^{33-35}$ Furthermore, a draft

\footnotetext{
${ }^{1}$ Department of Biosciences, University of Milan, Milan 20133, Italy; ${ }^{2}$ Plant Breeding, Wageningen University and Research, Wageningen $6700 \mathrm{AJ}, \mathrm{The}$ Netherlands; ${ }^{3}$ Research and Innovation Centre, Edmund Mach Foundation, San Michele all'Adige 38010, Italy; ${ }^{4}$ Biometris, Wageningen University and Research, Wageningen $6700 \mathrm{AA}$, The Netherlands; ${ }^{5}$ IRHS, INRA, AGROCAMPUS-Ouest, Université d'Angers, SFR 4207 QUASAV, Beaucouzé 49071, France; ${ }^{6}$ Department of Agricultural Sciences, University of Bologna, Bologna 40127, Italy;

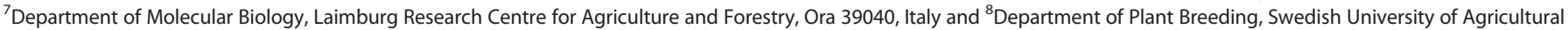
Sciences, Alnarp 23053, Sweden.

Correspondence: E van de Weg (eric.vandeweg@wur.nl)

${ }^{9}$ Current address: Hendrix Genetics Research, Technology \& Services, Boxmeer 5830 AC, The Netherlands.
}

Received: 7 October 2016; Revised: 25 October 2016; Accepted: 25 October 2016 
Table 1. Identity and origin of the 21 full-sib families used for developing the integrated genetic linkage map (iGLMap)

\begin{tabular}{|c|c|c|c|c|c|}
\hline Family & Mother & Father & Number of seedlings & Sources & Previous studies \\
\hline $12 \_B$ & 'Generos' & $X-6417$ & 48 & INRA_Angers-France & $13,41,71,72$ \\
\hline $12 \_E$ & 'Generos' & $X-6683$ & 58 & INRA_Angers-France & $13,41,71,72$ \\
\hline $12 \_\mathrm{F}$ & $X-3318$ & $X-6564$ & 48 & INRA_Angers-France & $13,41,71,72$ \\
\hline 12 & $x-3263$ & $X-3259$ & 47 & INRA_Angers-France & $13,41,71,72$ \\
\hline 12 J & $X-3318$ & 'Galarina' & 23 & INRA_Angers-France & $13,41,71,72$ \\
\hline $12 \_\mathrm{K}$ & $X-6679$ & $X-6808$ & 47 & INRA_Angers-France & $13,41,71,72$ \\
\hline $12 \_\mathrm{N}$ & $X-3305$ & $X-3259$ & 48 & INRA_Angers-France & $13,41,71,72$ \\
\hline $12+\mathrm{P}$ & 'Rubinette' & $x-3305$ & 48 & INRA_Angers-France & $13,41,71,72$ \\
\hline $\mathrm{Di} \overline{\mathrm{Pr}}$ & 'Discovery' & 'Prima' & 77 & JKI-Germany & $13,33,41,71-74$ \\
\hline DLO.12 & $1980-15-25$ & $1973-1-41$ & 219 & DLO-Netherlands & $27,75,76$ \\
\hline FuGa & 'Fuji' & 'Gala' & 141 & UNIBO-Italy & $41,71,77-79$ \\
\hline FuPi & 'Fuji' & 'Pinova' & 91 & RCL-Italy & $13,41,71,72$ \\
\hline $\mathrm{GaPi}$ & 'Gala' & 'Pinova' & 40 & RCL-Italy & $13,41,71,72$ \\
\hline I_BB & $X-6417$ & $X-6564$ & 43 & INRA_Angers-France & $13,41,71,72$ \\
\hline I_CC & $x-6679$ & 'Dorianne' & 50 & INRA_Angers-France & $13,41,71,72$ \\
\hline I_J & $\mathrm{X}-3318$ & $X-3263$ & 48 & INRA_Angers-France & $13,41,71,72$ \\
\hline I_M & $X-6683$ & $X-6681$ & 45 & INRA_Angers-France & $13,41,71,72$ \\
\hline I_W & $X-6398$ & $X-6683$ & 44 & INRA_Angers-France & $13,18,41,71,72$ \\
\hline JoPr & 'Jonathan' & 'Prima' & 174 & DLO-Netherlands & $13,71,72$ \\
\hline PiRea & 'Pinova' & 'Reanda' & 45 & JKI-Germany & $13,41,71,72$ \\
\hline $\mathrm{TeBr}$ & 'Telamon' & 'Braeburn & 202 & KUL-Belgium & $80-83$ \\
\hline Total & & & 1586 & & \\
\hline
\end{tabular}

These overview data have been partially presented by Bianco et $a l^{22}$ The number of genotyped seedlings has been updated after data curation in the current study during the construction of the iGLMap: 16 pairs of identical individuals were discovered across 6 families for which only 1 individual per pair was kept in the final data set; thus, a total of 16 identicals were removed. The involved families were 12 B (1 pair), DLO.12 (6 pairs), FuGa (1 pair), FuPi (1 pair), GaPi ( 3 pairs), I_M (1 pair), I_W (1 pair), JoPr (1 pair), and PiRea (1 pair). In addition, two individuals, 12_B058 and 12_J025 that showed a very high recombination rate ( $>5.0$ ) in almost all linkage groups were considered out-crossers and excluded from the final data set. Most of these populations were part of the previous European project HiDRAS, ${ }^{41}$ and four of them derived from other previous studies as reported in the last column. Pedigrees of the X-numbered accessions are given in Bink et al. ${ }^{13}$ a $\mathrm{X}-6690$.

apple reference genome sequence was available, ${ }^{36}$ which has been used for developing whole-genome genotyping (WGG) assays $^{22,36-38}$ for producing high-density SNP linkage maps on segregating FS families. ${ }^{28,34,35}$ However, all of the array and Genotyping By Sequencing (GBS) derived genetic linkage maps highlighted discrepancies in marker positions to the reference genome for $\sim 14$ (ref. 28) to $22 \%$ (ref. 32) of the markers. The generation of a highly reliable integrated map was prompted by the inconsistencies among these maps and by their low proportion of common markers.

Genetic linkage maps are created through the study of cosegregation patterns of markers and genes in segregating families. In outbreeding species, usually both parents can contribute segregation information and the generation of three different linkage maps is allowed: two parent specific maps and one integrated bi-parental map. A relevant issue in the construction of integrated genetic maps on bi-allelic markers, such as SNPs, is the high proportion of non-informative data, which are due to three main causes. First, missing values are inevitably high as most of the SNPs segregate in only one parent, thus being homozygous and not informative for the second parent. Second, markers segregating in both parents $(a b \times a b \rightarrow a a, a b, b b)$ yield only $50 \%$ of informative data since the alleles of the $a b$ progeny genotypes cannot be unequivocally traced back to the donor parent. The reduction in information is even worse when a null allele is present in both parental genotypes $(a-\times a-)$ : since their progeny is called by the presence $(a-$ and $a a)$ and absence $(--), 75 \%$ of the genotypes $(a-$ and $a a)$ cannot be unequivocally called and will be uninformative. Third, most markers usually do not segregate in each family. Therefore, the total amount of missing information goes well beyond $50 \%$ for any SNP marker. Uncertainty in marker order may also arise from standard approaches for map integration when merging the two parental maps of a FS family into a single bi-parental map through automated procedures. ${ }^{39,40}$ This process raises ambiguity in the appropriateness of marker order due to incompleteness in segregation information. Accordingly, linkage map integration across multiple bi-parental maps further increases ambiguities due to rise in missing data.

The main purpose of the present work was to produce a highly reliable and high-density integrated multi-parent genetic linkage map for apple (Malus domestica Borkh) to be used as a reference genetic map and as support in improving the apple genome assembly. To obtain the most reliable order for the highest possible number of markers, a novel mapping procedure was adopted by combining the following four main strategies: (i) using 21 segregating FS-families genotyped with the recent $20 \mathrm{~K}$ Infinium SNP array; ${ }^{22}$ (ii) reducing the proportion of noninformative data through an ad hoc SNP filtering and calling method and by the use of the HaploBlock (HB) bins formed by tightly linked markers; (iii) using a backcross (BC) design on singleparent data, rather than a Cross-Pollinator (CP) design on biparental ones, to facilitate the integration of parental data (full details are explained in the methods section); and (iv) using a twostep mapping procedure where an Initial Framework Map (IFM) of only the most informative markers, provides a reliable starting point for adding the remaining less informative.

\section{MATERIALS AND METHODS}

\section{Plant material}

This study included 1586 progeny plants from $21 \mathrm{FS}$ families. ${ }^{22}$ They were obtained from 26 parents and originated from six different breeding programs from five European countries (Table 1). Eighteen of them were part of the previous European project HiDRAS. ${ }^{41}$ Although most of the families comprised $\sim 50$ individuals, 7 of them significantly differed in size (Table 1), with 12 J being the smallest (23 individuals) and DLO.12 the largest (219 individuals). Part of these populations have also been used in studies on the development of multiplexes of SSR markers, ${ }^{42}$ validation of 
the pedigree-based analyses approach for QTL mapping on multiple pedigreed families ${ }^{13}$ and QTL discovery for horticultural traits. ${ }^{18}$

\section{DNA isolation}

For each individual, young, preferably unfolded leaves were sampled and freeze dried. Genomic DNA was extracted according to Schouten et al. ${ }^{27}$ The DNA was further purified using an RNAse step and quantified using $0.8 \%$ agarose gels and a dilution series of an external reference Lambda DNA (Invitrogen, Carlsbad, CA, USA).

\section{SNP-genotyping}

The 21 FS families and their parents were genotyped with the $20 \mathrm{~K}$ Infinium SNP array at the Fondazione Edmund Mach according to published procedures. ${ }^{28,37}$ SNP calling was performed using GenomeStudio software (Illumina Inc., San Diego, CA, USA; http://www. illumina.com), with a GenCall threshold of 0.15 , and ASSIsT, $^{43}$ a filtering and calling pipeline that accounts for the presence of null-alleles and signal intensity differences among $A B$-genotypes, thus increasing the number of usable SNP markers and providing some fully informative bi-parental markers with segregation type $a b \times a c$.

\section{SNP markers origin and focal point (FP) design}

The $20 \mathrm{~K}$ Infinium SNP array consists of three different SNP sources: the recently designed FruitBreedomics (FB) markers ${ }^{22}$ and subsets of the RosBREED SNPs and GD $\mathrm{Gnp}_{\text {sn }}$ markers (jointly referred to as IRSC-International Rosaceae SNP Consortium-markers) present on the previous $8 \mathrm{~K}$ Infinium SNP array. ${ }^{22,37}$ The $\mathrm{GD}_{\text {snp }}$ markers are based on polymorphisms within Golden Delicious sequence data, which were previously validated using SNPlex technology. ${ }^{36}$ The new FB markers were designed in clusters of up to 11 SNPs located within a region of at most $10 \mathrm{kbp}$, defined as Focal Point (FP) and distributed along the genome at distances of $\sim 1 \mathrm{cM}^{22}$ The $8 \mathrm{~K}$ Infinium SNP array also followed an FP design for the IRSC markers; however, here each FP stretched up to 100 kbp. $^{22,37}$

\section{Construction of bi-parental single-family linkage maps and SNP validation}

Before generating the integrated multi-parent linkage map, integrated biparental SNP linkage maps were created for each of the 21 FS-families to validate filtered SNPs and to verify the tight linkage between SNP markers coming from the same FP. For the construction of genetic linkage maps, JoinMap v4.1 (ref. 44) was used, applying the multipoint maximum likelihood mapping algorithm approach for cross pollinators ${ }^{40,45}$ and the Haldane mapping function using pre-set default settings. Markers were removed from the data set of an individual FS-family when they showed a severely distorted segregation $(P<0.01)$ and nearby markers segregating for the same parent did not show such a distortion, or when the rare genotype class occurred in less than $5 \%$ of the progeny. The GenomeStudio cluster plots were examined for the following: (i) markers mapping far (>10 cM) from any other marker; (ii) markers showing high nearest neighbor stress (NN stress) values ( $>2 \mathrm{cM}$ ) according to JoinMap output, and (iii) genotype calls involved in double recombinant singlepoints (singletons) as reported by JoinMap. When necessary and feasible, calls or the parental origin of markers were adjusted. Markers that remained problematic were excluded. Markers with identical genotypic scores (identicals), which are automatically set aside by JoinMap, were added back to the resulting linkage maps.

SNP markers from the same probe that mapped on different LGs in distinct families were classified as multi-locus SNPs, and they were considered as distinct markers specifying the mapping LG in their name. Also, the ' $\$$ ' symbol is added in front of their initial name to easily distinguish them from single-locus SNPs. Moreover, whenever SNP markers from the same FP were mapping to distinct genetic regions, the FP was split into two or more distinct SNP clusters, or in cases of an individual SNP, this was moved out of the FP. Finally, the assessed sets of co-segregating SNP markers belonging to the same FP were defined as HBs.

\section{SNP assignment to HBs}

Validated SNPs, as mapped in at least one of the 21 FS families (Supplementary Table S1), were grouped into HBs according to the FP design on the $20 \mathrm{~K}$ array ${ }^{22,37}$ or to their coordinates on the reference genome sequence v2 (in case of IRSC markers) ${ }^{22}$ while accounting for their co-segregation patterns in the individual $\mathrm{FS}$ families. $\mathrm{HBs}$ comprising only FB markers (FB-HBs) spanned at most $10 \mathrm{kbp}$, those including only IRSC markers (IRSC-HBs) covered at most $100 \mathrm{kbp}$ in size, and a window of $20 \mathrm{kbp}$ was assigned to HBs that comprised both FB and IRSC markers (FB + IRSC-HBs). Those SNPs that did not fall in any physical distance range allowed by the FP design were set aside the HBs and kept as individual SNP markers. Within each HB, SNP markers were ordered according to the coordinates of the targeted sequence polymorphism on the above mentioned genome sequence.

\section{The HB marker and the BC strategy}

The creation of $\mathrm{HBs}$ of co-segregating markers allowed a bin-mapping strategy where the segregation information of adjacent SNPs was aggregated and condensed into a single, virtual HB marker. The aggregation of co-segregating markers within the same HB increases the genotype score robustness consequently to information redundancy, and marker informativeness when combining markers with different segregation types. This is the case when a marker segregating in a single parent (for example, $a b \times a a$ ) is combined with a bi-allelic marker heterozygous in both parental plants (for example, $a b \times a b$ ) or with a single parent marker of the other sex (for example, $a a \times a b$ ), leading to the generation of a fully informative marker record (corresponding to a segregation type $a b \times a c$, or $a b \times c d$ ). In view of our mapping effort, this strategy was implemented in the ad hoc developed software Haploblock Aggregator (HapAg—http:// www.wageningenur.nl/en/show/HaploblockAggregator.htm) and applied to our data (Supplementary File S1). For each FS family, HapAg aggregated the segregation information of the SNP markers belonging to the same HB by using the information on linkage group and the linkage phase of the individual markers (Figure 1a), while considering the meiotic events occurring in the two parental plants separately. Thereto, HapAg splits the parental allelic contribution of every individual of each family into two distinct sub-data sets including either maternal or paternal recombination events (Figure 1b). Eventually, maternal and paternal data sets of all the progenies from all FS-families were merged to generate a single BC-type data set (Figure 1c), having twice the number of individuals as the original $\mathrm{CP}$ populations (a more detailed description of the methodological steps performed by HapAg is available in the software manual (http://www. wageningenur.nl/en/show/HaploblockAggregator.htm)). The BC segregation type allows the correct phasing of the markers segregating in different families, leading to integrating the genotypic data prior to map construction and to the production of a unique integrated genetic map rather than a map resulting from the a posteriori integration of the linkage maps obtained from FS families.

When inconsistencies between SNP scoring within the same HB are present (for example, due to recombination or gene conversion within the $\mathrm{HB}$ or calling issues), warning messages are reported, and the aggregated data score is set to missing (- ) by HapAg. The warnings were carefully examined to identify the origin of the issue and, when possible, to solve it before producing the final data set. Specifically, when conflicts occurred in more than five individuals per HB across all families, these were always inspected, while less consideration was given to a lower number of warnings as compatible to the expected number of true double recombination. Given the overall estimated genetic to physical size ratio of $548 \mathrm{kbp} / \mathrm{cm}$, based on the results from Velasco and colleagues, ${ }^{36}$ the probability of a recombination event occurring within a single $\mathrm{HB}$ is expected to be $1.8 \times 10^{-4}$ (FB-HBs), $1.8 \times 10^{-3}$ (IRSC - HBs) and $3.5 \times 10^{-4}$ $(\mathrm{FB}+\mathrm{IRSC}-\mathrm{HBs})$. These probabilities are so low that we decided to neglect such recombination, at least during the construction of the map, and thus, make missing the $\mathrm{HB}$ marker call for the potentially recombinant individual.

The final BC-type data set, formed by HB markers and individual SNPs not included in HBs (for simplicity, the generic term 'markers' will be used to refer to both), was used to construct the integrated Genetic Linkage Map (iGLMap).

\section{iGLMap construction}

Linkage maps were produced using JoinMap v4.1 with the same algorithm and parameter settings used when mapping the single FS-families, but now following a $B C$ design. Due to the sensitivity of mapping algorithms to missing data, ${ }^{40}$ a two-step mapping procedure was adopted. First, a reliable IFM was determined by including only markers that had genotypic data for at least $25 \%$ of the individuals (from 800 to $~ 3200$ available 


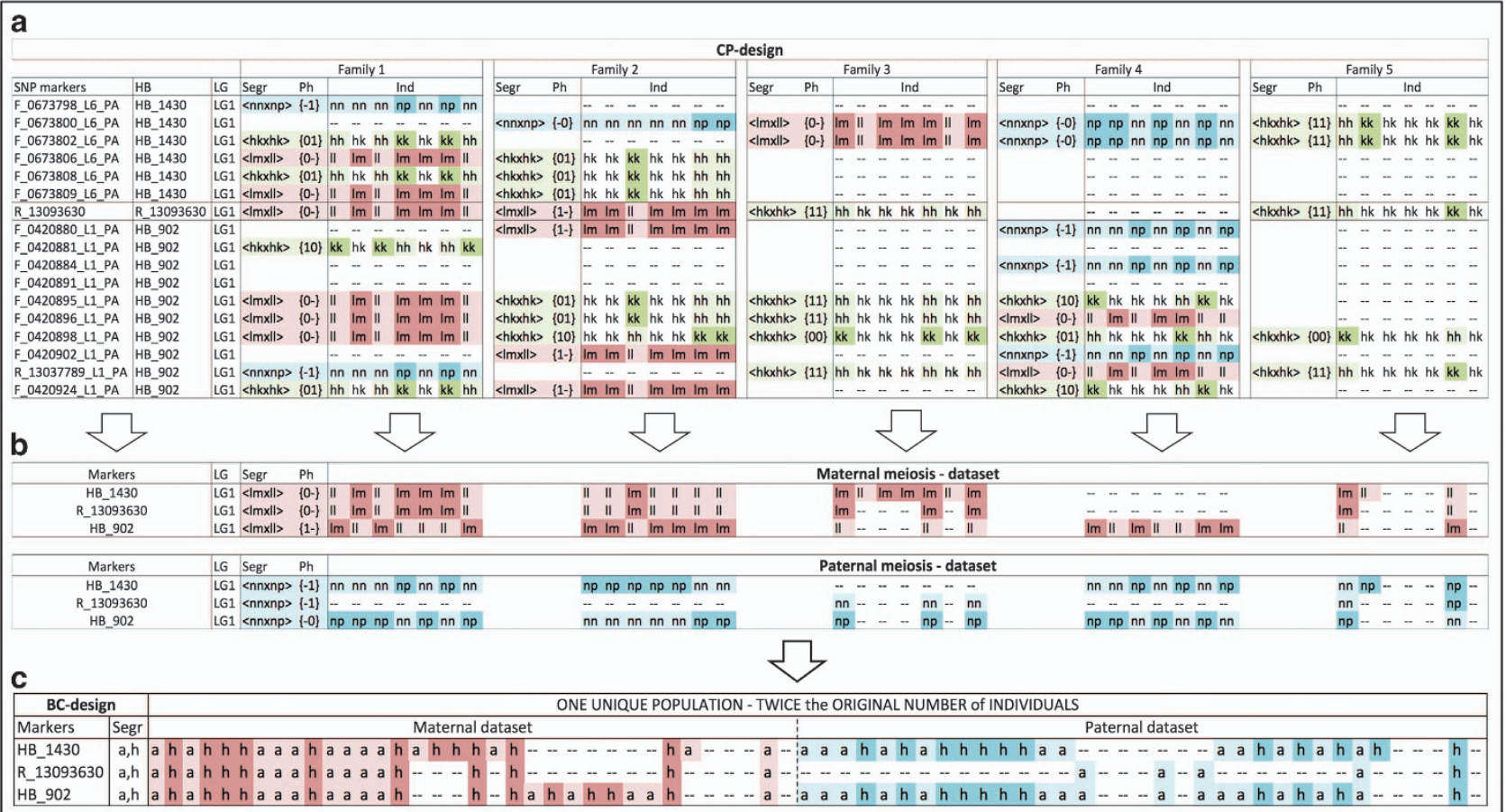

Figure 1. Graphical visualization of the combined HaploBlock and backcross approach presented in the current study. The figure illustrates the main steps of the process with an example from the true data of five families, each represented by seven individuals, two HaploBlocks (HBs) and one individual SNP on linkage group 1. Genotype codes presented here follow the format of JoinMap v3 and later versions for the crosspollinated (CP) segregation types (Segr), where $<\operatorname{Imxll}>$ refers to a maternal marker with genotypes Im and II, $<$ nnxnp $>$ to a paternal marker with genotypes $\mathrm{nn}$ and $\mathrm{np}$, and < hkxhk > refers to a bi-parental marker with genotypes hh, hk and kk (see https://www.kyazma.nl/ docs/JM4manual.pdf-Table 4). These three segregation types are highlighted with different colors: red for markers segregating only in the mother, blue for markers segregating only in the father, and green for those segregating in both parents; missing data (- -) and initially noninformative codes (hk) are not highlighted. (a) The use of the HB strategy allowed the identification of stable sets of SNP-markers, such as those composing HB_1430 and HB_902 that consist of 6 and 10 SNPs, respectively. These SNPs do not segregate in all families (the only exception is F_0420898_L1_PA), thus leading to a considerable amount of missing information (62\% of data points). (b) The genotypic information of the co-segregating SNPs is aggregated to form a single HB marker across families and the bi-parental allelic contribution is also split to form two distinct single-parent data sets, where the phase of the new 'single parent' HB-markers is adjusted accordingly. (c) The two complete single-parent data sets are subsequently converted in a backcross (BC) design and combined to form a unique population of twice the number of individuals as the initial CP populations. The presented strategy permits the almost complete exploitation of the segregation information available (losing only some information from the rare recombination events within a HB) while considerably reducing the amount of missing information: in this example, from $76 \%$ for the initial CP data sets of the two HBs to $28 \%$ in the final unique BC population. For the single SNP, the amount of missing data did not change throughout the process by definition and was $66 \%$. This approach of data aggregation and mating type was implemented in the software Haplotype Aggregator (HapAg-http://www.wageningenur.nl/en/show/HaploblockAg gregator.htm), whose manual describes the process in more detail.

meiosis). Subsequently, the map was completed by adding less informative markers with up to $90 \%$ missing data (from 320 to 800 available meiosis), while using the obtained IFM marker order as the Start Order in JoinMap. Markers with more than $90 \%$ missing values (327) were not considered. At both steps, for each linkage map, double recombinant single points (singletons) identified by the JoinMap Genotype probabilities function were visually examined through a graphical genotyping approach ${ }^{46}$ whereby data were displayed in map order as color-coded genotypes in Microsoft Excel using the conditional cell formatting feature. Issues causing singletons were investigated. For each $L G$, the best map was defined as the one with the lowest number of singletons.

\section{The iGLMap for apple genome improvement}

The produced IFM was used to examine and improve the reference apple genome sequence from $v 2$, as used for the design of the $20 \mathrm{~K}$ Infinium SNP array, ${ }^{22}$ to v3 (https://www.rosaceae.org/species/malus/malus_x_domes tica/genome_v3.0.a1). Specifically, this map helped to assess potential issues in the anchoring of contigs within scaffolds and provided a new assignment of scaffolds to chromosomes.

To confirm the collinearity between v3 of the reference genome and the final iGLMap, physical coordinates of all genetically mapped SNP markers were plotted as a function of genetic distances on the iGLMap. For comparison, collinearity between the iGLMap and the most widely used apple genome sequence $\mathrm{v} 1$ was also evaluated.
MareyMaps ${ }^{47}$ for each chromosome were produced using $R$ ( $R$ Development Team Core, Vienna, Austria, Europe).

\section{RESULTS}

Construction of 21 bi-parental linkage maps

The 21 FS families (Table 1) were genotyped using the $20 \mathrm{~K}$ Infinium SNP array. ${ }^{22}$ Overall, 15697 SNP markers (87\%) passed the SNP calling and filtering pipeline $\mathrm{ASSIST}^{43}$ and were genetically mapped using a final set of 1586 individuals. Singleparent map integration produced high-quality bi-parental maps for each family and LG (Table 2; Supplementary File S2), with six exceptions. LG6 and LG16 in family I_W and LG7 in family I_CC show large homozygous regions which led either to the absence of segregating bi-parental bridge markers (thus only single parent maps could be generated) (Supplementary Figures S1a and b), or to the lack of segregating markers in one parent (LG7 I_CC). Furthermore, LG17 in family I CC has no integrated map because the few bi-parental markers are not sufficiently spaced to allow orientation of the two single parent maps. This family is also peculiar since its paternal LG17 shows a highly distorted segregating region, coincident with the self-incompatibility 


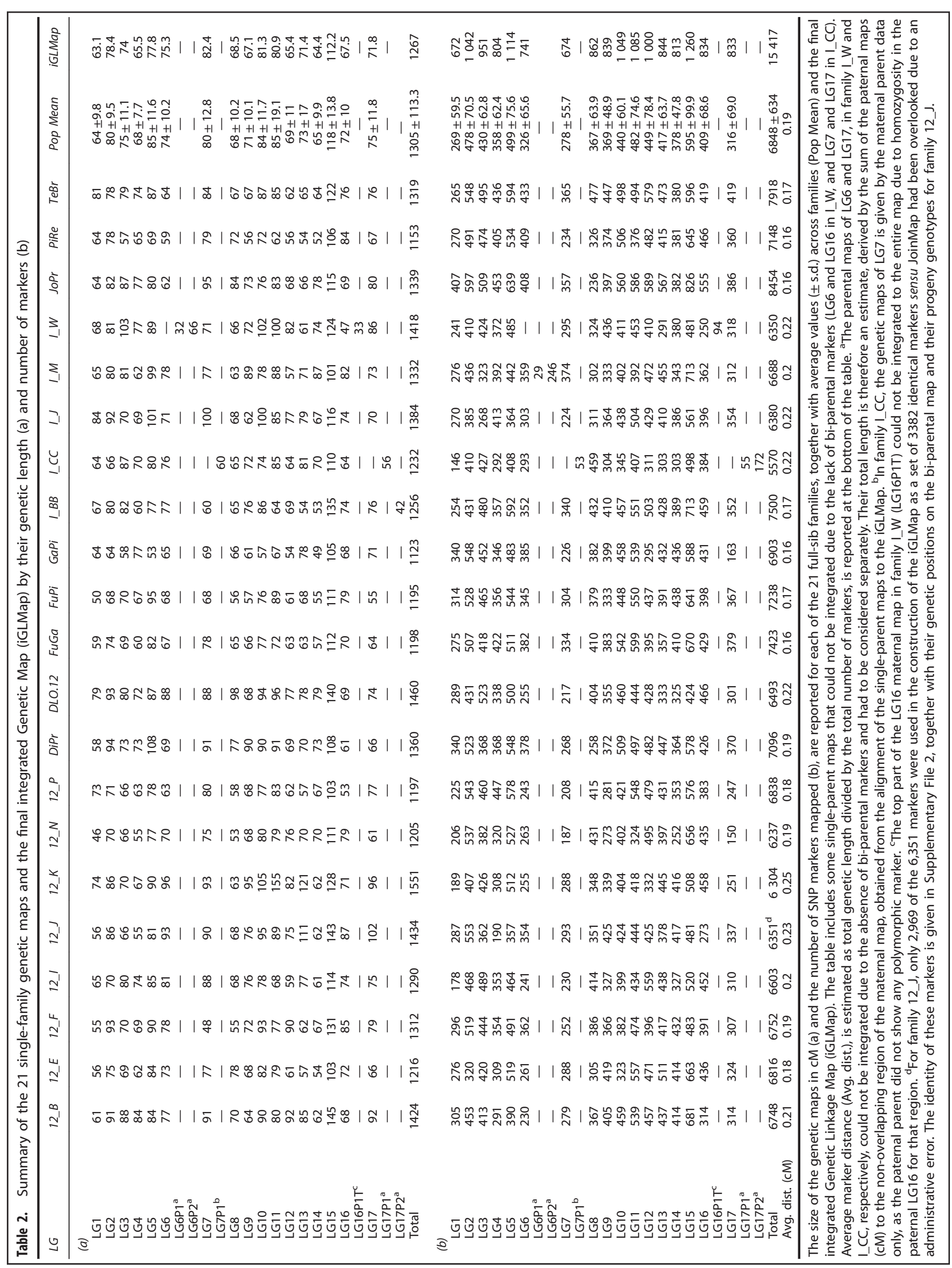


$S$ locus (Supplementary Figure S1c), thus suggesting the two parents to have a common self-incompatibility allele. Finally, the LGs 13 in families 12_J and 12_K have a genetic length of 1.5-1.6x the population mean (Table 2). Such relative large sizes are usually an indicator for data issues. This was true for 12_J, where the family size showed to be too small to come for a meaningful genetic map for this LG. For 12_K however we suspect a biological reason as its distorted segregations and double recombination pattern may be explained by the presence of natural selection at distant loci of the same chromosome (data not shown).

The total length of the 21 maps ranges from $1123(\mathrm{GaPi})$ to $1551 \mathrm{cM}\left(12 \_\mathrm{K}\right)$ with an average length of $1305 \mathrm{cM}( \pm 113.3)$ across families (Table 2a). The average distance between SNPs ranges from $0.16 \mathrm{cM}$ in JoPr to 0.25 in 12_K. On average across families, $6848( \pm 619)$ SNPs were mapped ranging from 5570 $\left(12 \_N\right)$ to 8454 (JoPr) (Table 2b).

Focal points validation and HBs identification

The order of markers on the 21 genetic maps was thoroughly checked, and correspondence to the apple's physical map v2 was assessed. Overall, the SNP marker order was coherent. Nonetheless, the following three main issues were identified that were common to all families and, overall, affected $22 \%$ of the markers: (i) inconsistencies in LG assignment for $17 \%$ of the markers; (ii) regions of inversion ( $\max \sim 2.5 \mathrm{Mbp}$ ) involving 3\% of the markers; and (iii) misplaced regions of markers within the same pseudochromosome for $2 \%$ of the markers. Furthermore, a close inspection of the bi-parental genetic maps highlighted the presence of 111 multi-locus SNPs (0.7\% of the markers), mapping in 2 or even 3 (on 4 occasions) different LGs across distinct families (Supplementary Table S2); 29 of these fall in known homoeologous regions originating from an ancient apple genome duplication. ${ }^{36}$ The 111 multi-locus SNP probes resulted in additional 115 alternative mapped SNP loci, establishing a final total number of 15812 validated SNP-loci, each segregating in at least one family (Table 3, Supplementary Table S1).

Therefore, the expected tight linkage between SNP markers of the same FP could not always be confirmed because 91 FPs included SNPs mapping to distinct genetic regions. Such discrepancies led to the creation of independent SNPs subclusters, identified as distinct HBs or individual SNPs. At this stage, 921 individual SNP markers and $2837 \mathrm{HBs}$ were considered (Table 4).

HB marker genotype definition and BC data set

The software HapAg was designed in the framework of the current mapping effort to aggregate the segregation information of the SNPs belonging to each HB into a single HB marker. During the aggregation process, HapAg reported 2848 inconsistencies in SNPs scoring within $748 \mathrm{HBs}(26 \%)$. More than half of them $(51 \%)$ resulted from the erroneous assignment of 55 SNPs to a FP and, therefore, to a HB, due to inadequate genome sequence information (unidentified during the mapping effort on single families). Those SNPs were removed from the HBs and used as individual SNP markers (Supplementary Table S1). The remaining $49 \%$ of conflicts involved $25 \%$ of the HBs and were due to calling errors, recombination within $\mathrm{HBs}$, or gene conversion.

The majority of these HBs (96.3\%) presented no more than 5 warnings, which were considered having a minor impact. The remaining 3.7\% reported between 6 and 16 conflicts. These inconsistencies were addressed by setting the $\mathrm{HB}$ score of the conflicting individual as missing. In fact, it would have been unfeasible to examine all cluster plots discriminating between calling errors, true recombination events, or gene conversion in a reasonable amount of time.

The final integrated data set consisted of a single BC-type population of 3172 individuals, including the genotypic information of 2837 HB makers and 976 individual SNPs. The overall proportion of missing information was massively reduced from $78 \%$ of the initial set of 15812 SNP markers to $54 \%$ of the final ( $\mathrm{HB}$ +individual SNPs) integrated data set (Figure 2), thus retaining the complete genetic information of the larger SNP data set.

\section{Construction of the iGLMap}

An IFM was constructed using markers that had data for at least $25 \%$ of the 3172 individuals, which was true for $2631 \mathrm{HB}$ and 344 individual SNP markers. The genetic length of its 17 LGs ranged from 64 (LG1) to $113 \mathrm{cM}$ (LG15), for a total IFM length of $1279 \mathrm{cM}$ (Table 5). The IFM was subsequently completed by adding markers with genotypic data for at least $10 \%$ of the individuals, to produce the iGLMap. Over the whole mapping process, an improvement in map quality was obtained through the close inspection of 1320 singletons by graphical genotyping (Supplementary File S3), whereby $67 \%$ of the singletons showed to result from 386 misplaced markers. These were moved to nearby positions. The adjusted marker order was then used as the Start Order for map re-estimation verifying that singletons' issues were indeed solved, and no new double recombinants were generated by the shift. Next, $13 \%$ of the singletons came from 13 HB markers that could not be adequately placed along the map, because they showed conflicting genotypes with adjacent markers at any position. Since the underlying cause could not be identified, those HB-markers (Supplementary Table S1) were removed. Finally, 20\% of the initial 1320 singletons remained. These are likely caused by genotyping errors, gene conversion events or true double recombinations. As the examination of each individual case would have been too time intensive, singletons' scores were set to missing, following Bassil et al. $^{48}$

The final iGLMap (Figure 3, Supplementary Figure S4) utilized 15417 SNPs (Table 3) and consisted of 3473 markers as follows: $2797 \mathrm{HBs}$ (Table 4), composed of a total of 14741 SNPs, and 676 individual SNP markers (Table 5). The total genetic length is $1267 \mathrm{cM}$, with LG1 being the shortest linkage group (63 cM) and LG15 being the longest (112 cM). The maximum distance between adjacent markers of $3.29 \mathrm{cM}$ is observed on LG6 (Table 5). An estimate of marker position robustness is provided by JoinMap

Table 3. Summary of the number of available, validated and mapped SNPs markers

\begin{tabular}{lccc}
\hline Source & $\begin{array}{c}\text { Available on the } \\
\text { 20 K array }\end{array}$ & $\begin{array}{c}\text { Validated } \\
\text { SNPs }\end{array}$ & $\begin{array}{c}\text { iGL mapped } \\
\text { SNPs }\end{array}$ \\
\hline FB & 14628 & 12508 & 12349 \\
Customized markers & 103 & 60 & 53 \\
IRSC-RosBREED & 2750 & 2601 & 2481 \\
IRSC-GDsnp & 538 & 528 & 520 \\
Multi-locus SNPs & & 115 & 14 \\
replicates & 18019 & 15812 & 15417 \\
Total & &
\end{tabular}

Abbreviations: FB, FruitBreedomics, designed in the $20 \mathrm{~K}$ Infinium SNP array development; ${ }^{22}$ IRSC, RosBREED and GDsnp markers, from the previously developed International Rosaceae SNP Consortium (IRSC) $8 \mathrm{~K}$ Infinium SNP array by Chagnè et al.; ${ }^{37}$ SNP, single-nucleotide polymorphism. Starting from the available markers as present on the $20 \mathrm{~K}$ Infinium SNP array, ${ }^{22}$ the number of validated SNPs (as the number of markers that mapped in at least one of the 21 individual families) and the number of SNP markers successful mapped on the integrated Genetic Linkage Map (iGL-mapped SNPs) are reported for each source type. Due to the presence of 111 multi-locus SNPs, the total count of validated and mapped SNPs includes multi-locus SNPs replicates as shown in the table. 


\begin{tabular}{|c|c|c|c|c|}
\hline $\begin{array}{l}\text { SNPs } \\
\text { source }^{\mathrm{a}}\end{array}$ & $\begin{array}{l}\text { Size } \\
(k b p)\end{array}$ & No. designed & No. mapped & $\begin{array}{c}\text { Max no. of clustered } \\
\text { SNPs }\end{array}$ \\
\hline FB & 10 & 1696 & 1670 & 11 \\
\hline $\mathrm{FB}+\mathrm{IRSC}$ & 20 & 642 & 640 & 15 \\
\hline IRSC & 100 & 499 & 487 & 8 \\
\hline Total & & 2837 & 2797 & \\
\hline \multicolumn{5}{|c|}{$\begin{array}{l}\text { Abbreviations: FB, FruitBreedomics; HB, HaploBlock; IRSC, International } \\
\text { Rosaceae SNP Consortium; SNP, single-nucleotide polymorphism. The } \\
\text { number of designed and mapped HBs is reported for each SNP source type } \\
\text { together with the physical distance range that defined the HB. The range } \\
\text { of clustered SNPs per HB went from two up to } 15 \text { SNPs (FB+IRSC markers). } \\
\text { aSee legend of Table } 3 \text {. }\end{array}$} \\
\hline
\end{tabular}

Plausible positions output table where, for each marker, the probability of its assigned and alternative positions are estimated across 1000 iterations. Graphical results for each LG are presented in Supplementary File S4. The order of markers appeared highly reliable, as all 1000 iterations resulted in a single position for most markers. Only a few regions show plausible alternative marker orders. Generally, such regions are characterized by very high marker density. When only two markers are involved, the distance between such markers is usually less than $0.01 \mathrm{cM}$. When three or more markers are involved, they usually span a region of less than $0.05 \mathrm{cM}$ (with the only exception of four regions in total, one on each of the linkage groups LG1, LG9, LG10 and LG15, which spanned from 0.07 up to $0.1 \mathrm{cM}$ ). Thus, the overall results show a robust and accurate marker order along the iGLMap with just a few small regions of uncertainty where the resolution was not enough to distinguish the genetic order of tightly linked markers.

\section{iGLMap collinearity with the apple genome}

The 21 bi-parental genetic maps already led to the identification of many regions where the genetic and physical maps deviate from collinearity. The final iGLMap allowed for visualization of inconsistencies with version $\mathrm{v} 1$ and $\mathrm{v} 3$ of the apple genome ${ }^{36}$ (Figure 4). The most used version is v1, which consists of a Primary Assembly and various alternative scaffold sequences (Figure 4a) representing homologous and possibly also some homoeologous sequences. Genetic data suggests the presence of some inverted regions that can be recognized by short lines at a right angle to the main curve of each graph in Figure $4 a$, which goes from the bottom-left to the top-right. Two examples are approximately at $20 \mathrm{cM}$ on LG10 and at $35 \mathrm{cM}$ on LG11. The short sequences of dots clearly aligned above or below the main curve represents regions where the position on the genome sequence may be shifted (that is, approximately at $80 \mathrm{cM}$ on LG10, at $75 \mathrm{cM}$ on LG11 and at $60 \mathrm{cM}$ on LG14). Some regions of the iGLMap have their sequence counterparts on non-corresponding pseudo-chromosomes. For example, the initial part of LG1 and the $22-34 \mathrm{cM}$ region of LG10 include markers belonging to other nine pseudo-chromosomes according to the genome sequence; also, the initial region of LG4 $(0-10 \mathrm{cM})$ consists almost entirely of markers from the pseudochromosome 9. Alternative scaffold sequences may also combine information from multiple pseudo-chromosomes. For example, the alternative scaffold on pseudo-chromosome 9 seems to contain, for the $20 \mathrm{Mb}$ region, a small fragment of its homoeologous region on LG17.

To support the development of a new $487 \mathrm{~K}$ Axiom array, ${ }^{38}$ version 3 of the apple reference genome was developed by using, among others, genetic information from the IFM. Adjustments were made only when entire contigs could be moved. Though overall collinearity has greatly improved, $2 \%$ of the genetically mapped

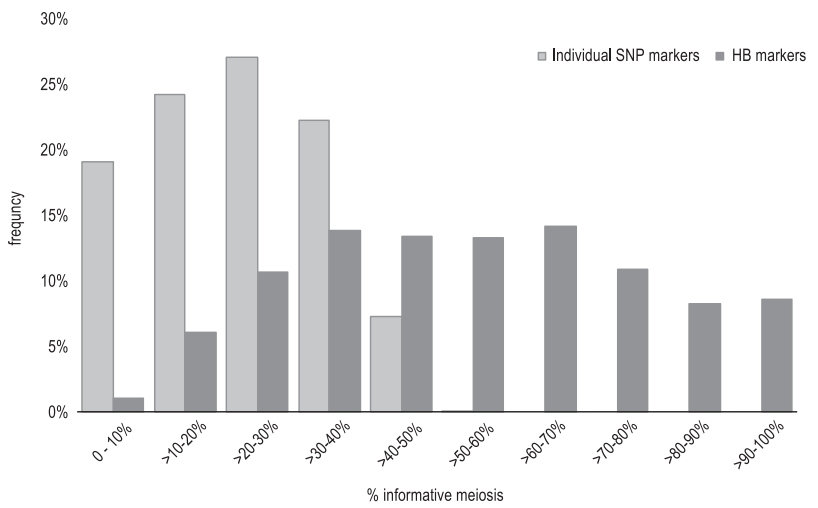

Figure 2. Frequency distribution of informative meiosis (in percentage) in the initial set of individual SNP markers (light gray bars) and the final set of HaploBlock (HB) together with remaining individual SNP markers (dark gray bars). The graph highlights the different amount of informative meiosis carried by individual SNPs and the more informative HB markers. SNP markers carried a maximum of $50 \%$ of the total information when being completely bi-allelic, as expected, and a maximum of $60 \%$ when being tri-allelic in some families when accounting for null-alleles and signal intensity differences. However, the latter is true only for a small proportion $(0.1 \%)$ of the SNPs, while the majority of SNPs is informative for 20$40 \%$ of the individuals. On the contrary, the majority of HB markers (+remaining single SNP) are informative for $40-80 \%$ of the individuals across all families and even $8.6 \%$ of the HBs is fully informative (100\%).

markers is not represented anymore, and inconsistency is still observed for $6 \%$ of the markers. As shown in Figure $4 \mathrm{~b}$, collinearity still varies considerably as follows: some LGs need further attention for just some narrow regions (for example, LG1 and LG17), while others have several regions to be ameliorated (for example, LG13 and LG16). At least six LGs show lines parallel to the main curve, suggesting the presence of scaffold sequence segments that have been misaligned (LG7, LG9, LG13, LG14, LG15 and LG16).

\section{DISCUSSION}

The iGLMap was developed through an innovative mapping approach whose power and major novelties rely on the use of HBs and a single BC-type data set. The HB approach provided highly informative common markers across families, facilitating integration of the maps. The use of the BC strategy applied to an outcrossing species allowed the integration of the genotypic data prior to map construction, avoiding the multiple efforts of parental map integration within and across FS families. The advantages of the HB strategy and BC design were strengthened by the use of an improved SNP filtering tool (ASSIsT ${ }^{43}$ ), which increased the number of informative markers; and by a multi-step map construction, which facilitated the inclusion of markers with relatively high missing value content in the final high-density linkage map.

\section{Use of multiple FS families}

The production of multifamily genetic maps can lead to an increase in marker density and genome coverage, overcoming local loss of genetic resolution and thus having the potential for a more accurate order of markers. ${ }^{49}$ To our knowledge, the iGLMap features the highest number of families ever used for a crop plant's genetic mapping, followed by 13 families for wheat. ${ }^{49}$ Indeed, the merging of data from 21 FS families resulted in an integrated map with an average of 2.3 times the number of markers of its preceding single family maps and a genetic length highly consistent with that of the preceding maps (284 cM shorter 
Table 5. Final integrated Genetic Linkage Map (iGLMap) and core map (values in brackets), summarized per linkage group (LG) by number of HB-markers, genetic length, average and maximum interval between adjacent markers

iGLMap (core map)

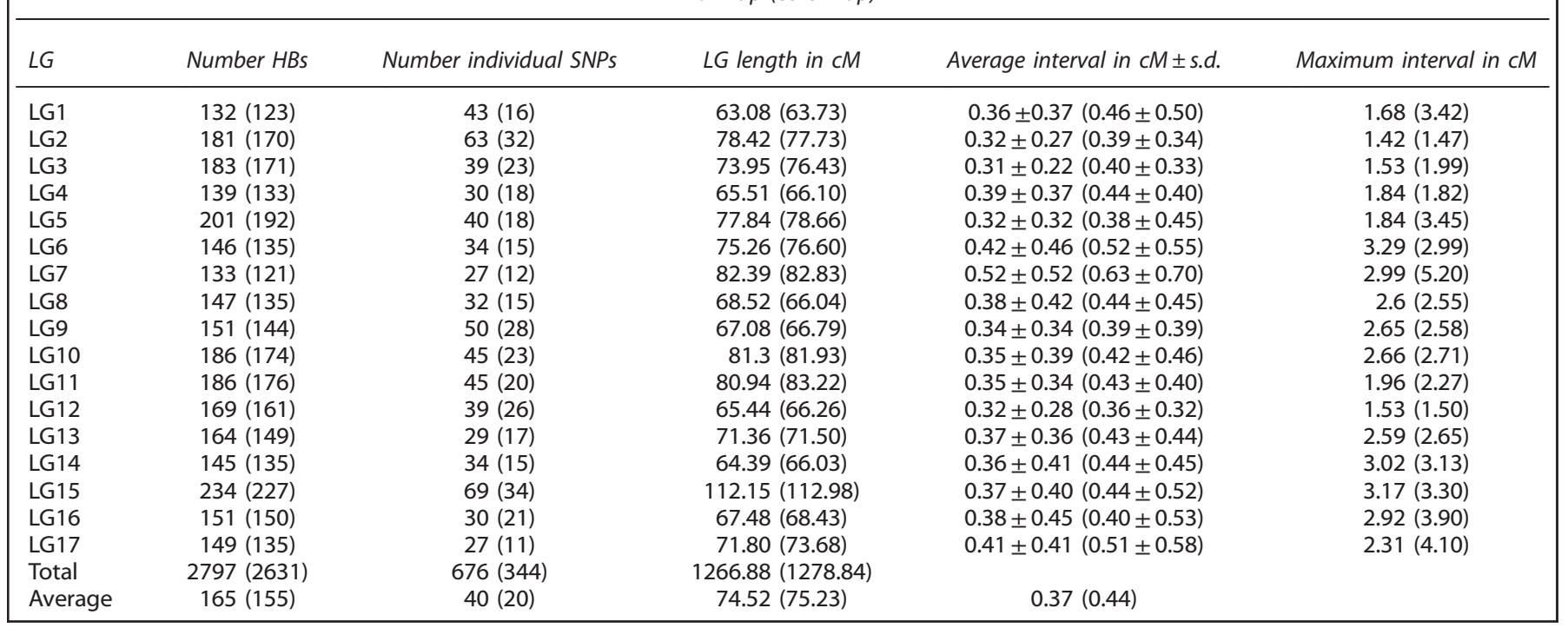

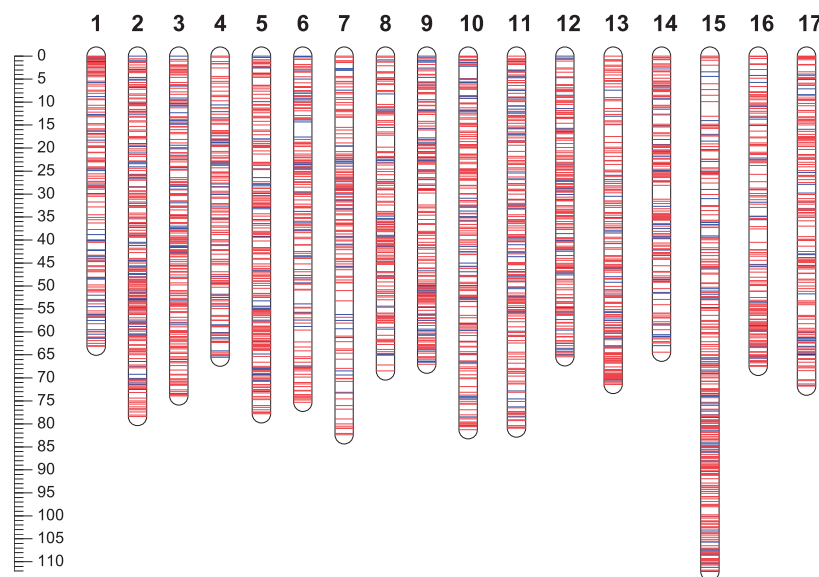

Figure 3. Visual presentation of the distribution of the iGLMap haploblock markers (red lines) and single SNP markers (blue lines) over the 17 linkage group apple. Position and name of the markers is detailed in Supplementary Figure S3.

than the longest and $144 \mathrm{cM}$ longer than the shortest one). The enhanced map resolution is indicated by the consistent reduction in the maximum distance between adjacent markers as follows: $3.29 \mathrm{cM}$ on the LG6 of the iGLMap, compared to values for individual families ranging from 5.94 (LG7 of the FuGa family) to 30.14 cM (LG13 of the 12_K family). The use of multiple families thus allowed for overcoming the limitations that occurred in single families including issues related to large regions of homozygosity and to extremely skewed segregation ratios, as those reported here for families I_W and I_CC, and solved in the final iGLMap.

\section{Use of improved SNP calling and HBs}

The essence of our strategy is the reduction of missing information, which usually hampers accurate marker ordering. Therefore, we aimed to include as many common SNPs as possible by exploiting information from null-alleles, and to increase information content of bi-parental $a b \times a b$ markers also by exploiting signal intensity differences. ${ }^{43,50}$ This contrasts with standard SNP calling procedures, which usually do not recognize null-alleles and discard such markers while classifying them as 'non-Mendelian'.

The use of null-alleles is expected to improve mapping efficiency not only by increasing the number of common markers, but also by increasing information content of single SNP markers: this allows the use of tri-allelic markers of type $a 0 \times b 0$ that provide full segregation information for both parents. ${ }^{43,50}$ Accounting for differences in signal intensity due to an additional SNP at the probe site also gives rise to fully informative markers, because two variants may occur from one or both of the marker alleles converting bi-allelic $a b \times a b$ markers into tri-allelic $a b \times a b^{\prime}$ ones. ${ }^{43,50}$

Our data set included 1244 SNP markers with null-alleles that segregated in at least one FS-family, resulting in additional data for a total of 1974 marker/family combinations. Of these, 327 markers resulted in $494(25 \%)$ combinations that showed full segregation information $(a b \times a c), 514$ markers out of $915(46 \%)$ combinations that segregated as dominant $a b \times a b^{\prime}$ markers for which a null-allele was present in both parents. Additionally, 328 initial $a b \times a b$ markers became fully informative $a b \times a c$ markers for 471 combinations by exploiting signal intensity differences resulting from an additional SNP at the probe site. The accounting for null-alleles and for differences in signal intensity thus increased the amount of segregation information for HB-markers and individual SNP-markers. The impact of this method on the final data set will vary with the study population and will increase at decreasing numbers of families and HB size.

The proposed HB strategy may show some similarities with bin mapping, although it presents substantial differences that can lead to a more accurate marker positioning. Usually, bin mapping is based on the identification of an interval (bin) along a linkage group where, given a small set of individuals, no recombination events are observed for any of them. ${ }^{51}$ This approach has been very successful to obtain approximate genetic information on marker position along a chromosome using a small number of individuals. ${ }^{52-54}$ However, the smallest unit of resolution in these 
a

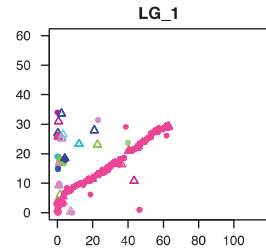

LG_7

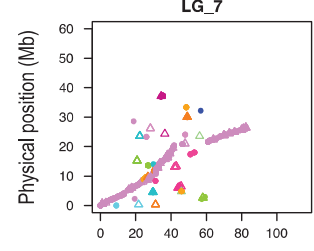

LG_13

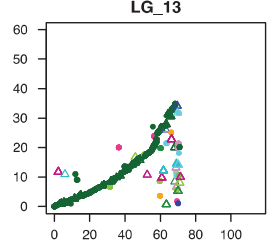

b

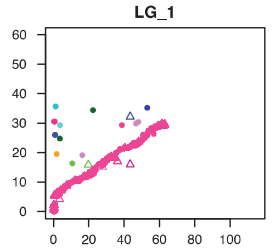

LG_7

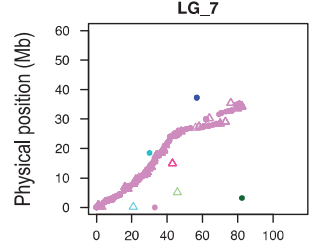

LG 13

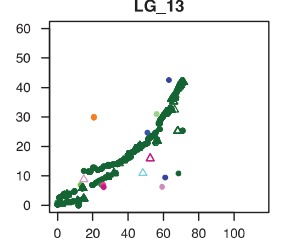

LG_2

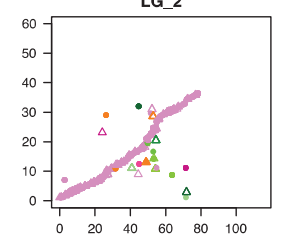

LG_8

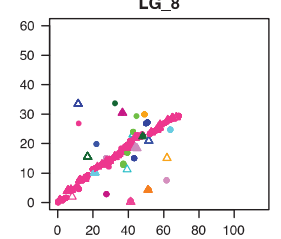

LG_14

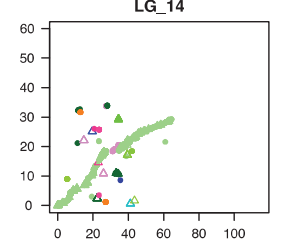

LG_2

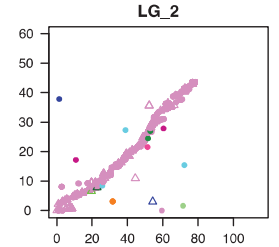

LG 8

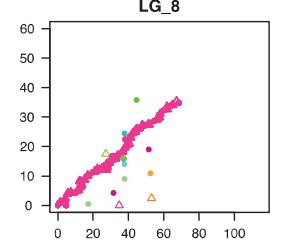

LG_14

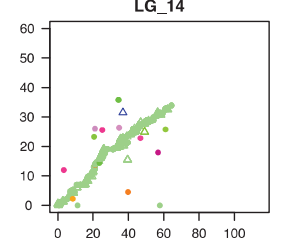

LG_3

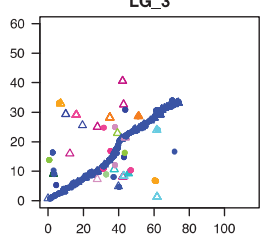

LG_9

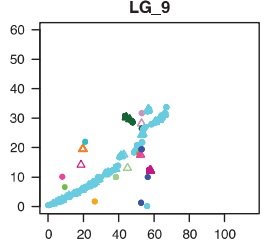

LG_15

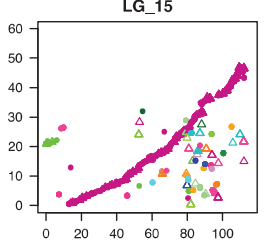

LG_4

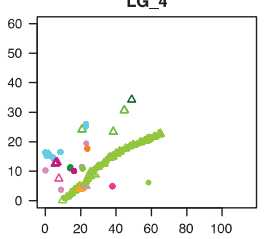

LG_10

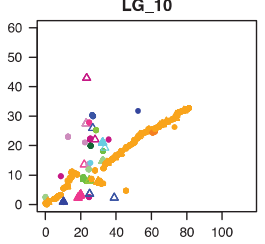

LG_16
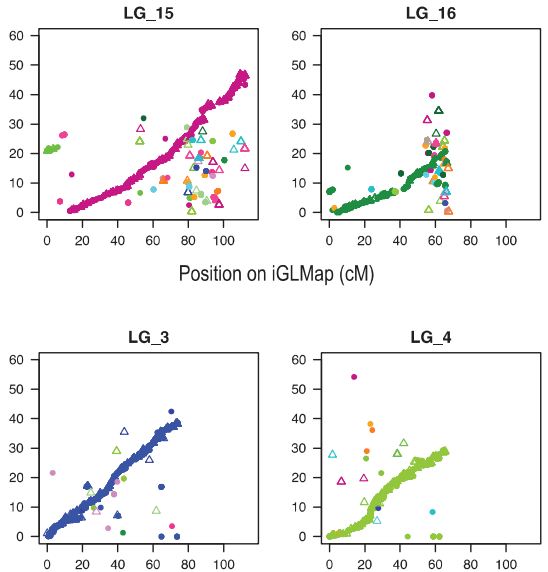

LG_9

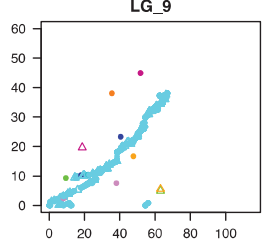

LG_15

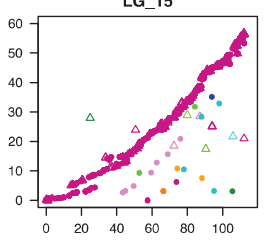

LG_4

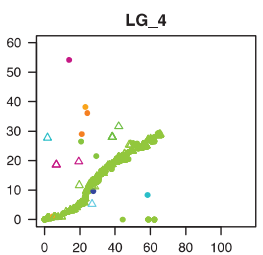

LG_10

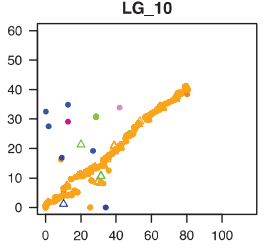

LG_16

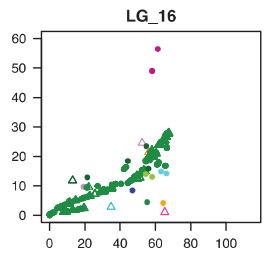

LG_5

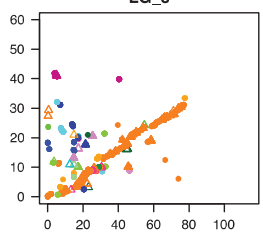

LG_11

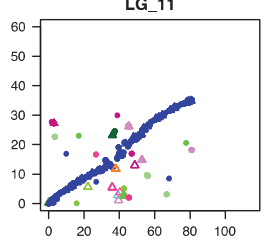

LG_17

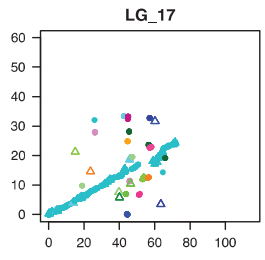

LG_5

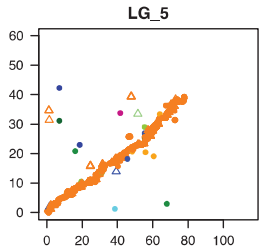

LG_11

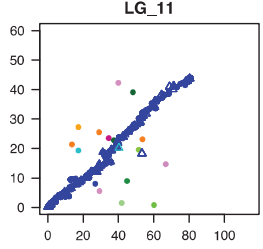

LG_17

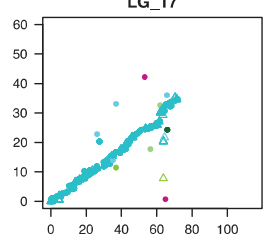

LG_6

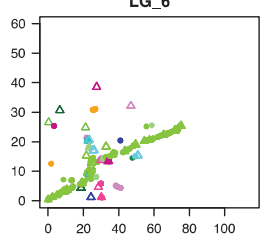

LG_12

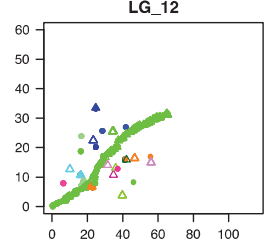

Figure 4. Collinearity between the integrated Genetic Linkage Map (iGLMap) and v1 (a) and v3 (b) of the apple genome sequence. Physical coordinates for SNP markers were retrieved from v1 (15 133 SNP markers traced) and v3 (14 539 SNP markers traced) of the Apple Genome and their physical positions $(\mathrm{Mb})$ are plotted versus marker genetic positions (cM). Filled dots and triangles indicate markers physically

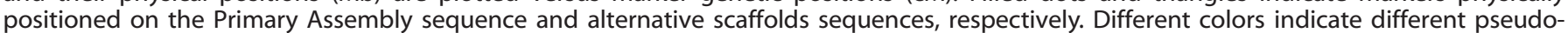
chromosomes according to the legend in the figure; thus, markers inconsistently assigned to Linkage Groups (LGs) and pseudo-chromosome are immediately highlighted.

genetic maps was given by the bin, whose length usually spanned from an average size of $7.8 \mathrm{cM}$ up to a maximum length of $33 \mathrm{cM} .{ }^{52,54}$ Conversely, our HB strategy defined bins based on (very short) physical distances. The use of multiple SNPs within a narrow physical bin was anticipated in the design of the 8 and $20 \mathrm{~K}$ Infinium SNP arrays through the identification of tightly clustered SNP markers within a small physical window. ${ }^{22,37}$

Indeed, the HB strategy was very effective as it led to an increase in marker robustness through the exploitation of redundant genetic information of adjacent markers, and to an increase in information content, especially when markers were of different segregation type. In fact, in pseudo-testcross analysis, ${ }^{39}$ bi-allelic markers heterozygous in both parents lead to $50 \%$ noninformative data as it is not possible to unequivocally establish the origin of the alleles in the heterozygous progenies. On the contrary, the proposed HB strategy is able to exploit these data to produce a robust and fully informative HB marker when such biparental markers are combined with markers of other segregation type(s) within the same HB (see family 2 in Figure 1). Consequently, whereas all single bi-allelic SNP markers were informative 
for less than $50 \%$ of the progenies across multiple families (Figure 3), 55\% of the aggregated HB-markers are informative for more than $50 \%$ of the individuals, and $8.6 \%$ of the HB-markers presented 90 to $100 \%$ of the information, thus providing highly informative bridge markers common to most FS-families. The use of HB-markers thus favored map integration across families and allowed high accuracy in marker position along the chromosome.

In this study, we did not further examine within-HB SNPs order, and we disregarded a priori any recombination inside HBs by making the relative marker scores missing. However, the within$\mathrm{HB}$ recombinations may still be useful in the future to validate local genome assemblies when needed. We estimate that $\sim 1940$ within $\mathrm{HB}$ recombinations will be present in the whole data set. This estimation is based on a genome-wide relationship between physical and genetic distances of $586 \mathrm{~Kb} / \mathrm{cM}$ (the estimated genome size of $742 \mathrm{Mb}$ [ref. 36] divided by the length of the iGLMap), the physical length covered by the HBs (using the number of mapped HBs and their maximum allowed size for each of the three different HB-type), the total number of individuals (3 172), the availability of informative data (46\%), and assuming absence of crossover interference.

This estimated number is certainly inflated, as crossover interference does occur. Furthermore, the distance between the two most apart and informative SNPs within a HB is less than the allowed maximum $\mathrm{HB}$ size. Also, not all these recombinations will be noticed as having occurred within the $\mathrm{HB}$, because, in many parents, HBs lack the two or more informative SNPs needed to observe recombination. Consequently, the number of expected observable recombination events might not be very different from the 1396 conflicts observed among SNP calls within HBs. Notably, if such estimations are correct, they also imply the absence of major calling issues in the final data set.

The HB approach makes use of all the available markers, including identical markers sensu JoinMap. These are markers that have exactly identical genotype calls across all progenies of a single mapping family, including an identical linkage phase. In the analysis of single families JoinMap removes such identicals from the data set by default, thus reducing memory requirements and computation time. However, we re-entered them because they are needed for the construction of HB markers. The availability of full genetic information for each $\mathrm{HB}$, completed by the use of the identicals, allows the adequate mapping of these HBs. Furthermore, in the case of conflicting data within a HB, the presence of identical markers may be helpful in tracing the inconsistency.

\section{BC mapping strategy}

The most commonly used linkage analysis approach for outbreeding FS families is based on the two-way pseudo-testcross strategy, ${ }^{39}$ which results in the production of two single-parent maps to be then integrated using the available bi-parental bridging markers. Despite the advances in map integration methods ${ }^{40,55-58}$ the process is still based on computing the average of marker distances over the two parental maps, which is affected by the different segregation types and by the number and informativeness of bridging bi-parental markers. The consequent result is a loss of parental-specific features, low accuracy in marker order ${ }^{4}$ and inconsistencies between individual maps. Similarly, the most popular approaches used in the generation of multi-population consensus maps ${ }^{59-61}$ are based on the integration of marker distances over populations. Therefore, map integration is not a straightforward process, especially because usually not all the loci are common in all populations, and additional factors, such as the local reordering and marker misplacement, further affect resolution and accuracy of the consensus map.

On the contrary, the proposed BC design, leads to the generation of a single data set where genotypic data have been merged prior to the generation of integrated genetic maps. This strategy reduces the integration process to a single step where segregation data, recombination events and marker order are directly related and accessible for close inspection across all germplasm through visual approaches, such as graphical genotyping. ${ }^{46}$ The use of this strategy to obtain high-quality linkage maps is demonstrated by the short length of the iGLMap and the robustness of its marker order (see below). Moreover, the use of a condensed, fully informative data set optimizes computational performance.

A multi-step mapping procedure and data curation through visual inspection

Multi-step procedures are very often used for the construction of high-density consensus maps as they allow for marker order optimization and genotyping error correction. ${ }^{62}$ In standard mapping approaches, bi-parental segregating framework markers can be used to produce back-bone maps, which provide a fixed order on which the final consensus map is built by adding the remaining markers. ${ }^{63}$ Bin-mapping methods also follow a twophase mapping process based on the initial construction of highconfidence framework maps to which new markers are subsequently added. ${ }^{25,51,64}$

In our case, the choice to pursue a two-step mapping procedure was further motivated by the sensitivity of the JoinMap mapping algorithms to the presence of missing data. ${ }^{40}$ In fact, JoinMap was not able to perform the grouping when analyzing all 3473 markers simultaneously. For this reason, we first decided to produce a robust IFM including only the most informative markers.

Nevertheless, in high-density marker data sets, the marker order proposed by JoinMap demonstrated sensitivity to the presence of single problematic data points ${ }^{48}$ and/or structured missing data as present in our final data set due to markers segregating in some families but not in others. This may lead to inadequate marker orders that can cause inflation of map size, a high number of singletons and larger regions of double recombination. In Bassil et al., ${ }^{48}$ as in our current study, the use of a graphical genotyping tool was crucial to perform adequate data curation through identifying problematic data points and alternative marker orders for improving the map quality and reducing singletons and double recombination.

\section{Evaluation of map quality}

High quality and robustness of the iGLMap is inferred from its genetic length when compared to other published linkage maps of apple. Since the release of the genome sequence of apple $e^{36}$ and the consequent extensive development of whole genome genotyping SNP arrays, ${ }^{22,37}$ several high-density genetic maps of apple were constructed. Most of them are integrated bi-parental maps of one family, where the number of individuals ranges from 118 to 297, comprising different type of markers (for example, SNP +SSR for a total of 1,016 markers; ${ }^{34}$ SNP+SSR for a total of 2579 markers; ${ }^{28}$ SNP+SSR for a total of 601 markers; ${ }^{65} 3441$ SNP (ref. 66)) and GBS based linkage maps comprising 3967 SNP (ref. 29), 1053 SNP (ref. 31), and 1918 and 2818 SNP markers (ref. 32). Furthermore, a consensus map of apple was produced by merging a SNP genetic map with maps from earlier studies on eight FS families ${ }^{34}$ as follows: two based on SSR markers ${ }^{24,25}$ and six based on SSR and SNP markers. ${ }^{36}$ This consensus map consisted of 2875 markers with a total genetic length of $1991 \mathrm{cM}$. Finally, a multifamily single parent map was produced for the 'Honeycrisp' apple from three small progenies (318 individuals in total) having 1091 SNPs (ref. 35). All of these genetic maps have a total size ranging from $1282-1991 \mathrm{cM} .{ }^{28,34}$ The iGLMap is shorter than all these despite having the highest chance of false recombination due to data issues; and therefore an inflated genetic length due to being based on the largest number of markers, the largest number 
of FS families and the largest total number of individuals. This result once more proves the quality of the iGLMap and thereby the validity of our approach.

Further support for the quality of the iGLMap comes from the plots of the alternative plausible positions calculated by JoinMap. In the iGLMap, spots with uncertain marker order usually spanned less than $0.05 \mathrm{cM}$, and only occasionally spanned up to $0.1 \mathrm{cM}$. Such limited uncertainty was feasible thanks to the reduced level of missing values, the high level of data curation, and the high number of individuals that were included in our study, which theoretically allow a maximum resolution of $\sim 0.02 \mathrm{cM}$ when two $\mathrm{HB}$ markers segregate in all individuals.

The length of the iGLMap (1267 cM) is shorter than both the direct and weighted average length of the 21 single family maps (1 $305 \mathrm{cM}$ ), because additional data curation had been performed after the construction of the individual maps, during the data aggregation and the mapping of the obtained HB data, as described in the result section.

Proposal for a re-orientation of the LG5 linkage map

In apple and pear, the convention for orienting linkage groups and pseudo-molecules is based on the genetic linkage map by Maliepaard et $a l^{2}{ }^{2}$ for 'Prima' $x^{\prime}$ 'Fiesta'. Maliepaard et $a l^{2}$ were the first to report large stretches of homoeologous sequences as detected by multi-locus targeting RFLP clones. However, they did not use these first indications on homoeology for linkage group orientation. To date, those initial observations have been confirmed, and knowledge about duplication patterns have considerably increased. ${ }^{36}$ In apple, the orientation of the two chromosomes of the homoeologous pairs 5 and 10 and 13 and 16, where homoeology involves almost the entire chromosome, showed opposite orientation sensu Maliepaard et al. $^{2}$ The orientation of LG13 has been modified to match that of LG16 by Liebhard et al. ${ }^{67}$ In the present work, we modified the orientation of the LG5 linkage map to match that of LG10 to facilitate future chromosome and genome comparison studies between homoeologous chromosomes within a species and synteny studies among species. We re-orientated LG5 and not LG10 because LG5 has been reported in less genetic studies on QTL and candidate genes identification in apple (Supplementary File S5). Due to the limited number of cases where putative QTLs and genes have been reported on LG5, we expect the benefits for future studies to offset the initial inconvenience generated by comparing our iGLMap with those of previous papers. In pear, the naming and orientation of linkage groups was based on apple ${ }^{68}$ having made use of the high synteny between these two crops. ${ }^{68,69}$ In the few QTL reports on pear, LG5 has been mentioned more frequently than LG10 (Supplementary File S5).

The HB strategy, genotyping by sequencing and single families The HB strategy contributed considerably to the quality of the IGLMap. This strategy is applicable to a wide range of genetic data and population types. Indeed, in this study, HB-sizing was essentially based on information from a draft genome sequence: Haploblock markers were created by the aggregation of segregation information from SNP that co-localized within a narrow physical window. The approach is also applicable with GBS derived data. In case of, Restriction site Associated DNA (RAD) sequencing, ${ }^{70}$ the various SNP from the same read may be used for the construction of a HB marker. The HB strategy may also be used on single families as it may considerably reduce computation time, especially in case of a large sized family and very high marker densities.
Conclusion

The reliable marker order, high coverage and resolution of the iGLMap makes it a valid reference map for QTL mapping studies and the evaluation of genome assemblies. Therefore, the iGLMap can contribute to the enhancement of marker-assisted breeding approaches aimed at improving apple quality and productivity. The usefulness of the iGLMap has already been demonstrated in at least the following two occasions: i) its successful use in a recent pedigree-based analyses study identifying QTLs and possibly the underlying candidate genes for controlling chilling and heat requirements ${ }^{18}$ and ii) the improvement of the reference genome sequence of apple (https://www.rosaceae.org/species/malus/ malus_x_domestica/genome_v3.0.a1).

Finally, the methodology presented here may be valuable for the construction of accurate high-density bi- and multi-parental integrated genetic linkage maps for any outbreeding species.

\section{CONFLICT OF INTEREST}

The authors declare no conflict of interest.

\section{ACKNOWLEDGEMENTS}

We thank Yolanda Noordijk for the isolation of DNA from all samples at WageningenUR and Elisa Banchi for her work on the genotyping of these samples with the $20 \mathrm{~K}$ Infinium SNP array at the Fondazione Edmund Mach. This work has been co-funded by the EU seventh Framework Programme by the FruitBreedomics project $\mathrm{N}^{\circ}$. 265582: Integrated Approach for increasing breeding efficiency in fruit tree crops (www.FruitBreedomics.com). The views expressed in this work are the sole responsibility of the authors and do not necessary reflect the views of the European Commission

\section{REFERENCES}

1 Gianfranceschi L, Koller B, Seglias N, Kellerhals M, Gessler C. Molecular selection in apple for resistance to scab caused by Venturia inaequalis. Theor Appl Genet 1996; 93: 199-204.

2 Maliepaard C, Alston FH, van Arkel G, Brown LM, Chevreau E, Dunemann F et al. Aligning male and female linkage maps of apple (Malus pumila Mill.) using multi-allelic markers. Theor Appl Genet 1998; 97: 60-73.

3 Gygax M, Gianfranceschi L, Liebhard R, Kellerhals M, Gessler C, Patocchi A. Molecular markers linked to the apple scab resistance gene $\mathrm{Vbj}$ derived from Malus baccata jackii. Theor Appl Genet 2004; 109: 1702-1709.

4 Bartholomé J, Mandrou E, Mabiala A, Jenkins J, Nabihoudine I, Klopp C et al. High-resolution genetic maps of Eucalyptus improve Eucalyptus grandis genome assembly. New Phytol 2015; 206: 1283-1296.

5 Nakamura S, Asakawa S, Ohmido N, Fukui K, Shimizu N, Kawasaki S. Construction of an $800-k b$ contig in the near-centromeric region of the rice blast resistance gene Pi-ta 2 using a highly representative rice BAC library. Mol Gen Genet 1997; 254: 611-620

6 Yang D, Sanchez A, Khush GS, Zhu Y, Huang N. Construction of a BAC contig containing the xa5 locus in rice. Theor Appl Genet 1998; 97: 1120-1124.

7 Vinatzer BA, Patocchi A, Gianfranceschi L, Tartarini S, Zhang H-B, Gessler C et al. Apple Contains Receptor-like Genes Homologous to the Cladosporium fulvum resistance gene family of tomato with a cluster of genes cosegregating with $\mathrm{vf}$ apple scab resistance. Mol Plant Microbe Interact 2001; 14: 508-515.

8 Lande R, Thompson R. Efficiency of marker-assisted selection in the improvement of quantitative traits. Genetics 1990; 124: 743-756.

9 Asíns MJ. Present and future of quantitative trait locus analysis in plant breeding Plant Breed 2002; 121: 281-291.

10 Laurens F, Aranzana MJ, Arús P, Bassi D, Bonany L, Corelli L et al. Review of fruit genetics and breeding programmes and a new European initiative to increase fruit breeding efficiency. Acta Hort 2012; 929: 95-102.

11 Scossa F, Brotman Y, de Abreu e Lima F, Willmitzer L, Nikoloski Z, Tohge T et al. Genomics-based strategies for the use of natural variation in the improvement of crop metabolism. Plant Sci 2016; 242: 47-64.

12 Bink MCA. M, Boer MP, ter Braak CJF, Jansen J, Voorrips RE, van de Weg WE. Bayesian analysis of complex traits in pedigreed plant populations. Euphytica 2007; 161: 85-96.

13 Bink MC A. M, Jansen J, Madduri M, Voorrips RE, Durel C-E, Kouassi $A B$ et al. Bayesian QTL analyses using pedigreed families of an outcrossing species, with application to fruit firmness in apple. Theor Appl Genet 2014; 127: 1073-1090. 
14 Rosyara UR, Bink MCAM, van de Weg E, Zhang G, Wang D, Sebolt A et al. Fruit size QTL identification and the prediction of parental QTL genotypes and breeding values in multiple pedigreed populations of sweet cherry. Mol Breed 2013; 32: 875-887.

15 Fresnedo-Ramírez J, Bink MCAM, van de Weg E, Famula TR, Crisosto $\mathrm{CH}$, Frett TJ et al. QTL mapping of pomological traits in peach and related species breeding germplasm. Mol Breed 2015; 35: 166.

16 Fresnedo-Ramírez J, Frett TJ, Sandefur PJ, Salgado-Rojas A, Clark JR, Gasic K et al. QTL mapping and breeding value estimation through pedigree-based analysis of fruit size and weight in four diverse peach breeding programs. Tree Genet Genomes 2016; 12: 25.

17 Roach JA, Verma S, Peres NA, Jamieson AR, van de Weg WE, Bink MCAM et al. FaRXf1: a locus conferring resistance to angular leaf spot caused by Xanthomonas fragariae in octoploid strawberry. Theor Appl Genet 2016; 129: 1191-1201.

18 Allard A, Bink MCAM, Martinez S, Kelner J-J, Legave J-M, Di Guardo M et al. Detecting QTLs and putative candidate genes involved in budbreak and flowering time in an apple multiparental population. J Exp Bot 2016; 67: 2875-2888.

19 Jannink J-L. Identifying Quantitative trait locus by genetic background interactions in association studies. Genetics 2007; 176: 553-561.

20 Huang X, Paulo M-J, Boer M, Effgen S, Keizer P, Koornneef $M$ et al. Analysis of natural allelic variation in Arabidopsis using a multiparent recombinant inbred line population. Proc Natl Acad Sci USA 2011; 108: 4488-4493.

21 Huang BE, Verbyla KL, Verbyla AP, Raghavan C, Singh VK, Gaur P et al. MAGIC populations in crops: current status and future prospects. Theor Appl Genet 2015; 128: 999-1017.

22 Bianco L, Cestaro A, Sargent DJ, Banchi E, Derdak S, Di Guardo M et al. Development and validation of a $20 \mathrm{~K}$ Single Nucleotide Polymorphism (SNP) whole genome genotyping array for apple (Malus $\times$ domestica Borkh). PLOS ONE 2014; 9: e110377.

23 Hemmat M, Weeden NF, Brown SK. Mapping and evaluation of Malus $\times$ domestica microsatellites in apple and pear. J Am Soc Hortic Sci 2003; 128: 515-520.

24 Silfverberg-Dilworth E, Matasci CL, van de Weg WE, Kaauwen MPWV, Walser M, Kodde LP et al. Microsatellite markers spanning the apple (Malus $\times$ domestica Borkh.) genome. Tree Genet Genomes 2006; 2: 202-224.

25 Celton J-M, Tustin DS, Chagné D, Gardiner SE. Construction of a dense genetic linkage map for apple rootstocks using SSRs developed from Malus ESTs and Pyrus genomic sequences. Tree Genet Genomes 2008; 5: 93-107.

26 Han Y, Zheng D, Vimolmangkang S, Khan MA, Beever JE, Korban SS. Integration of physical and genetic maps in apple confirms whole-genome and segmental duplications in the apple genome. J Exp Bot 2011; 62: 5117-5130.

27 Schouten HJ, van de Weg WE, Carling J, Khan SA, McKay SJ, Kaauwen MPW et al. Diversity arrays technology (DArT) markers in apple for genetic linkage maps. Mol Breed 2011; 29: 645-660.

28 Antanaviciute L, Fernández-Fernández F, Jansen J, Banchi E, Evans KM, Viola R et al. Development of a dense SNP-based linkage map of an apple rootstock progeny using the Malus Infinium whole genome genotyping array. BMC Genomics 2012; 13: 203.

29 Gardner KM, Brown P, Cooke TF, Cann S, Costa F, Bustamante C et al. Fast and cost-effective genetic mapping in apple using next-generation sequencing. $G 3$ (Bethesda) 2014; 4: 1681-1687.

30 Falginella L, Cipriani G, Monte C, Gregori R, Testolin R, Velasco R et al. A major QTL controlling apple skin russeting maps on the linkage group 12 of 'Renetta Grigia di Torriana'. BMC Plant Biol 2015; 15: 150.

31 Ban SH, Lee SJ, Choi C. Development of an apple f1 segregating population genetic linkage map using genotyping-by-sequencing. Agrotechnology 2016; 5: 2.

32 McClure KA, Gardner KM, Toivonen PM, Hampson CR, Song J, Forney CF et al. QTL analysis of soft scald in two apple populations. Hortic Res 2016; 3: 16043.

33 N'Diaye A, van de Weg WE, Kodde LP, Koller B, Dunemann F, Thiermann M et al. Construction of an integrated consensus map of the apple genome based on four mapping populations. Tree Genet Genomes 2008; 4: 727-743.

34 Khan MA, Han Y, Zhao YF, Troggio M, Korban SS. A multi-population consensus genetic map reveals inconsistent marker order among maps likely attributed to structural variations in the apple genome. PLOS ONE 2012; 7: e47864.

35 Clark MD, Schmitz CA, Rosyara UR, Luby JJ, Bradeen JM. A consensus 'Honeycrisp' apple (Malus $\times$ domestica) genetic linkage map from three full-sib progeny populations. Tree Genet Genomes 2014; 10: 627-639.

36 Velasco R, Zharkikh A, Affourtit J, Dhingra A, Cestaro A, Kalyanaraman A et al. The genome of the domesticated apple (Malus $\times$ domestica Borkh.). Nat Genet 2010; 42: 833-839.

37 Chagné D, Crowhurst RN, Troggio M, Davey MW, Gilmore B, Lawley C et al. Genome-wide SNP detection, validation, and development of an $8 \mathrm{~K}$ SNP array for apple. PLOS ONE 2012; 7: e31745.
38 Bianco L, Cestaro A, Linsmith G, Muranty H, Denancé C, Théron A et al. Development and validation of the Axiom Apple480K SNP genotyping array. Plant J 2016; 86: 62-74.

39 Grattapaglia D, Sederoff R. Genetic linkage maps of Eucalyptus grandis and Eucalyptus urophylla using a pseudo-testcross: mapping strategy and RAPD markers. Genetics 1994; 137: 1121-1137.

40 Van Ooijen JW. Multipoint maximum likelihood mapping in a full-sib family of an outbreeding species. Genet Res 2011; 93: 343-349.

41 Gianfranceschi L, Soglio V. The European project HiDRAS: innovative multidisciplinary approaches to breeding high quality disease resistant apples. Acta Hortic 2004; 663: 327-330.

42 Patocchi A, Fernández-Fernández F, Evans K, Gobbin D, Rezzonico F, Boudichevskaia A et al. Development and test of 21 multiplex PCRs composed of SSRs spanning most of the apple genome. Tree Genet Genomes 2008; 5: 211-223.

43 Di Guardo M, Micheletti D, Bianco L, Putten HJJK, Longhi S, Costa F et al. ASSIsT: An automatic SNP scorlng tool for in- and outbreeding species. Bioinformatics 2015, btv446.

44 Van Ooijen JW. JoinMap 4, Software for the Calculation of Genetic Linkage Maps in Experimental Populations, In: Kyazma BV: Wageningen, The Netherlands. 2006.

45 Jansen RC, Jannink J-L, Beavis WD. Mapping quantitative trait loci in plant breeding populations. Crop Sci 2003; 43: 829-834.

46 Young ND, Tanksley SD. Restriction fragment length polymorphism maps and the concept of graphical genotypes. Theor Appl Genet 1989; 77: 95-101.

47 Rezvoy C, Charif D, Guéguen L, Marais GAB. MareyMap: an R-based tool with graphical interface for estimating recombination rates. Bioinformatics 2007; 23: 2188-2189.

48 Bassil NV, Davis TM, Zhang H, Ficklin S, Mittmann M, Webster T et al. Development and preliminary evaluation of a $90 \mathrm{~K}$ Axiom SNP array for the allo-octoploid cultivated strawberry Fragaria $\times$ ananassa. BMC Genomics 2015; 16: 155.

49 Maccaferri M, Ricci A, Salvi S, Milner SG, Noli E, Martelli PL et al. A high-density, SNP-based consensus map of tetraploid wheat as a bridge to integrate durum and bread wheat genomics and breeding. Plant Biotechnol J 2015; 13: 648-663.

50 Pikunova A, Madduri M, Sedov E, Noordijk Y, Peil A, Troggio M et al. 'Schmidt's Antonovka' is identical to 'Common Antonovka', an apple cultivar widely used in Russia in breeding for biotic and abiotic stresses. Tree Genet Genomes 2014; 10: 261-271.

51 Vision TJ, Brown DG, Shmoys DB, Durrett RT, Tanksley SD. Selective mapping: a strategy for optimizing the construction of high-density linkage maps. Genetics 2000; 155: 407-420.

52 Howad W, Yamamoto T, Dirlewanger E, Testolin R, Cosson P, Cipriani G et al. Mapping with a few plants: using selective mapping for microsatellite saturation of the Prunus reference map. Genetics 2005; 171: 1305-1309.

53 Sargent DJ, Cipriani G, Vilanova S, Gil-Ariza D, Arús P, Simpson DW et al. The development of a bin mapping population and the selective mapping of 103 markers in the diploid Fragaria reference map. Genome 2008; 51: 120-127.

54 Fernandez-Silva I, Eduardo I, Blanca J, Esteras C, Picó B, Nuez F et al. Bin mapping of genomic and EST-derived SSRs in melon (Cucumis melo L.). Theor Appl Genet 2008; 118: 139-150.

55 Jansen J, de Jong AG, van Ooijen JW. Constructing dense genetic linkage maps. Theor Appl Genet 2001; 102: 1113-1122.

56 Wu R, Ma C-X, Wu SS, Zeng Z-B. Linkage mapping of sex-specific differences. Genet Res 2002; 79: 85-96.

$57 \mathrm{Wu}$ R, Ma C-X, Painter I, Zeng Z-B. Simultaneous maximum likelihood estimation of linkage and linkage phases in outcrossing species. Theor Popul Biol 2002; 61: 349-363.

58 Jansen J. Construction of linkage maps in full-sib families of diploid outbreeding species by minimizing the number of recombinations in hidden inheritance vectors. Genetics 2005; 170: 2013-2025.

59 Stam P. Construction of integrated genetic linkage maps by means of a new computer package: Join Map. Plant J 1993; 3: 739-744.

$60 \mathrm{Wu}$ Y, Close TJ, Lonardi S. Accurate construction of consensus genetic maps via integer linear programming. IEEE/ACM Trans Comput Biol Bioinform 2011; 8: 381-394.

61 Ronin Y, Mester D, Minkov D, Belotserkovski R, Jackson BN, Schnable PS et al. Two-phase analysis in consensus genetic mapping. G3 GenesGenomesGenetics 2012; 2: 537-549.

62 Liu D, Ma C, Hong W, Huang L, Liu M, Liu H et al. Construction and analysis of high-density linkage map using high-throughput sequencing data. PLOS ONE 2014; 9; e98855.

63 Hedgecock D, Shin G, Gracey AY, Berg DVD, Samanta MP. Second-generation linkage maps for the pacific oyster Crassostrea gigas reveal errors in assembly of genome scaffolds. G3 (Bethesda) 2015; 5: 2007-2019.

64 Avni R, Nave M, Eilam T, Sela H, Alekperov C, Peleg Z et al. Ultra-dense genetic map of durum wheat $\times$ wild emmer wheat developed using the $90 \mathrm{~K}$ iSelect SNP genotyping assay. Mol Breed 2014; 34: 1549-1562. 
65 Ma B, Zhao S, Wu B, Wang D, Peng Q, Owiti A et al. Construction of a high density linkage map and its application in the identification of QTLs for soluble sugar and organic acid components in apple. Tree Genet Genomes 2015; 12: 1-10.

66 Sun R, Chang Y, Yang F, Wang Y, Li H, Zhao Y et al. A dense SNP genetic map constructed using restriction site-associated DNA sequencing enables detection of QTLs controlling apple fruit quality. BMC Genomics 2015; 16: 747.

67 Liebhard R, Kellerhals M, Pfammatter W, Jertmini M, Gessler C. Mapping quantitative physiological traits in apple (Malus $\times$ domestica Borkh.). Plant Mol Biol 2003; 52: $511-526$.

68 Yamamoto T, Kimura T, Terakami S, Nishitani C, Sawamura Y, Saito T et al. Integrated reference genetic linkage maps of pear based on SSR and AFLP Markers. Breed Sci 2007; 57: 321-329.

69 Yamamoto T, Terakami S. Genomics of pear and other Rosaceae fruit trees. Breed Sci 2016; 66: 148-159.

70 Miller MR, Dunham JP, Amores A, Cresko WA, Johnson EA. Rapid and cost-effective polymorphism identification and genotyping using restriction site associated DNA (RAD) markers. Genome Res 2007; 17: 240-248.

71 Kouassi AB, Durel C-E, Costa F, Tartarini S, van de Weg E, Evans K et al. Estimation of genetic parameters and prediction of breeding values for apple fruit-quality traits using pedigreed plant material in Europe. Tree Genet Genomes 2009; 5: 659-672.

72 Muranty H, Troggio M, Sadok IB, Rifaï MA, Auwerkerken A, Banchi E et al. Accuracy and responses of genomic selection on key traits in apple breeding. Hortic Res 2015; 2: 15060.

73 Dunemann F, Ulrich D, Boudichevskaia A, Grafe C, Weber WE. QTL mapping of aroma compounds analysed by headspace solid-phase microextraction gas chromatography in the apple progeny 'Discovery' $x^{\prime}$ Prima'. Mol Breed 2009; 23: 501-521.

74 Dunemann F, Boudichevskaia A, Grafe C. QTL and candidate gene mapping for aroma compounds in the apple progeny 'Discovery' $\times$ 'Prima'. Acta Hortic 2009; 839: $433-440$.

75 Soriano JM, Joshi SG, van Kaauwen M, Noordijk Y, Groenwold R, Henken B et al. Identification and mapping of the novel apple scab resistance gene $V d 3$. Tree Genet Genomes 2009; 5: 475-482.
76 Soriano JM, Madduri M, Schaart JG, van der Burgh A, van Kaauwen MPW, Tomic L et al. Fine mapping of the gene Rvi18 (V25) for broad-spectrum resistance to apple scab, and development of a linked SSR marker suitable for marker-assisted breeding. Mol Breed 2014; 34: 2021-2032.

77 Costa F, Stella S, van de Weg WEV, Guerra W, Cecchinel M, Dallavia J et al. Role of the genes Md-ACO1 and Md-ACS1 in ethylene production and shelf life of apple (Malus domestica Borkh). Euphytica 2005; 141: 181-190.

78 Costa F, Peace CP, Stella S, Serra S, Musacchi S, Bazzani M et al. QTL dynamics for fruit firmness and softening around an ethylene-dependent polygalacturonase gene in apple (Malus $\times$ domestica Borkh.). J Exp Bot 2010; 61: 3029-3039.

79 Costa F, Cappellin L, Farneti B, Tadiello A, Romano A, Soukoulis C et al. Advances in QTL mapping for ethylene production in apple (Malus $\times$ domestica Borkh.). Postharvest Biol Technol 2014; 87: 126-132.

80 De Wit I, Cook NC, Keulemans J. Characterization of tree architecture in two-yearold apple seedling populations of different progenies with a common columnar gene parent. Acta Hortic 2004; 663: 363-368.

81 Kenis K, Keulemans J. Genetic linkage maps of two apple cultivars (Malus $\times$ domestica Borkh.) based on AFLP and microsatellite markers. Mol Breed 2005; 15: 205-219.

82 Davey MW, Kenis K, Keulemans J. Genetic control of fruit vitamin C contents. Plant Physiol 2006; 142: 343-351.

83 Kenis K, Keulemans J, Davey MW. Identification and stability of QTLs for fruit quality traits in apple. Tree Genet Genomes 2008; 4: 647-661.

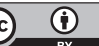

This work is licensed under a Creative Commons Attribution 4.0 International License. The images or other third party material in this article are included in the article's Creative Commons license, unless indicated otherwise in the credit line; if the material is not included under the Creative Commons license, users will need to obtain permission from the license holder to reproduce the material. To view a copy of this license, visit http://creativecommons.org/licenses/ by/4.0/

(c) The Author(s) 2016

Supplementary Information for this article can be found on the Horticulture Research website (http://www.nature.com/hortres) 